\title{
TREATMENT OF GLEET.
}

\author{
BY A. H. HARKNESS, M.R.C.S., L.R.C.P. \\ Hon. Medical Officer in Charge, V.D. Department, St. Peter's Hospital.
}

BEFORE commencing a course of treatment, the cause of the gleet must be accurately determined, and the lesion or lesions found treated on orthodox lines. It is almost useless to make a "shot in the dark," and at one visit give a prostatic massage and at the next a Kollmann.

If an attack of acute gonorrhœa is not cured by the sixth week, it may be said to have passed from the acute to the chronic stage and, in investigating such cases, I suggest the following routine examination : Examine the patient after he has held his urine for five hours, or preferably before he has passed his urine first thing in the morning. Smears should be taken of the discharge, also cultures, and lesions, such as infected para-urethral glands or ducts, should be looked for. The next thing to determine is whether the infection is anterior or posterior. This is usually done by the two-glass test, but in my opinion this is generally useless in cases of gleet, as the amount of discharge is so small that the first gush of urine washes it into the first glass, and it is impossible to say whether it comes from the anterior or posterior urethra.

A more accurate method is the following: Before the patient urinates, wash out the anterior urethra thoroughly with a lukewarm lotion at a low head of pressure $(2 \mathrm{ft}$.) until the washings come away clear with no threads. Then the patient should urinate, and if the urine is hazy or contains threads, the presence of a posterior urethritis may be assumed. A digital examination should then be made to detect any abnormalities of the prostate, vesiculæ seminales, or Cowper's glands. Only gross abnormalities can be detected by the finger. After massage of the organs, smears of the urethral discharge and the urine passed should be examined microscopically for pus cells and organisms.

Air distension urethroscopy is the most useful method for examining the anterior urethra. Great care should be taken in using these instruments to prevent the possibility of air embolism. If there is any blood on the swab used to remove the lubricant, it is a wise plan to postpone the examination for a week. Catastrophes usually occur when there is a laceration in the region of a hard stricture. The examination should be carried out as quickly as possible ; the tube should not fit too tightly, and the urethra should not be over-distended with air.

With the anterior urethroscope one can diagnose a littritis, soft or hard strictures, lesions of Cowper's ducts on the floor of the bulbous urethra, and, occasionally, signs of over-treatment. The most serious error in the diagnosis of chronic gleet is to overlook a urethral stricture.

The following are the commonest causes of gleet: Prostatitis, vesiculitis, cowperitis, littritis, soft and hard infiltrations, infection of the para-urethral glands or ducts, chemical urethritis and urethritis due to over-instrumentation. There are other more serious lesions of the urinary tract which may give rise to a urethral discharge and so simulate a gleet, and when in doubt, a complete investigation, including an X-ray examination, should be made. In these cases the urethritis descends from the upper urinary tract, as in cases of calculus pyonephrosis, stone in the bladder, ureter, prostate or urethra, or in 
infections of the urinary tract with Bacillus coli or other pyogenic organisms. I have seen three cases where the so-called gleet was tubercular in origin, and also one case which was proved conclusively to be one of bilharzia hæmatobia.

In certain cases where local treatment has been carried out for many months without a rest, it is advisable to stop all treatment for a fortnight, and if the patient is only suffering from a urethritis due to over-treatment, marked improvement will soon be noticed. A discharge is often provoked by treatment, and a complete rest will give the urethra an opportunity of recovering from a chemical urethritis or one that is due to over-instrumentation. During this rest I usually prescribe a mixture containing ol. santal flav. $15 \mathrm{~m}$. t.d.s. Even if the condition does not entirely clear up with a cessation of all local treatment, there will be marked improvement in a gleet as soon as orthodox treatment is recommenced.

It is essential to carry out posterior irrigations during the treatment of gleet, although it is seldom necessary to do them more than once a day. Potassium permanganate $1 / 7,000$ to $1 / 4,000$ is the solution of choice in the treatment of acute gonorrhœa, but in the treatment of gleet, especially where the infection is posterior, I find that silver nitrate $\mathrm{I} / 20,000$ to $\mathrm{I} / \mathrm{IO}, 000$ most useful. During a course of instrumentation, also when the infection is found to be due to non-specific organisms, oxycyanide of mercury $\mathrm{I} / 6,000$ to $1 / 4,000$ gives the best results.

For the treatment of littritis and soft infiltrations, the flushing Kollmann is preferable. Massage over large straight sounds gives good results, but the meatus will not always admit a large instrument, and it then becomes necessary to use mechanical dilators. The Kollmann should not be used more often than every five or six days, and at each subsequent sitting the dilatation should, if possible, be increased. A local anæsthetic? should not be used, and as soon as the patient experiences slight pain, the dilatation should not be proceeded with at that sitting, as any trauma to the urethral mucous membrane does more harm than good. If there are infiltrations on the roof of the bulb in the region of the opening of the middle urethra, the passing of large curved sounds is more efficacious than the Kollmann.

For the treatment of hard strictures, I dilate up to I8 F with Eynard's soft bougies, and then carry on the dilatations with steel sounds. After each sitting the urethra should be irrigated with $1 / 4,000$ oxycyanide of mercury.

Digital massage of the prostate per rectum once or twice a week is the most effective method of treatment for chronic prostatitis. It empties into the prostatic urethra the accumulated contents of the gland, increases the blood-supply to the part, and at the same time gives the patient an autogenous vaccine. In view of this fact, it is not wise to give a vaccine on the same day, as the reaction may be too great. Before carrying out this treatment the bladder should be filled with lotion, and for chronic prostatitis I prefer to use silver nitrate $\mathrm{I} / 20,000$ to $\mathrm{I} / \mathrm{10}, 000$. After massage, the patient should pass the lotion, which will wash away both the debris in the urethra and that which has found its way into the bladder. The massage of the organ should be done as gently as possible, because, if undue force is used, it is an easy matter at any stage of a prostatitis to precipitate an epididymitis. Janet considers that massage of the prostate has caused many more complications in the form of epididymitis than it has cured cases of prostatitis. Epididymitis should be a very rare complication, and is usually due to indiscretions on the part of the patient or to the faulty technique of the surgeon. If 
there is an acute attack of epididymitis, all local treatment should be stopped for about a fortnight. The patient should go to bed and have hot boracic fomentations applied frequently to the scrotum. Large steels are also a useful adjunct in the treatment of chronic prostatitis, but they should never be passed more often than once a week.

The treatment of chronic vesiculitis is similar to that of chronic prostatitis, and after a little practice it is an easy matter to strip the vesiculæ seminales of their contents. By gentle palpation with the pulp of the finger, one very seldom fails to palpate the normal vesicle, but one does not always feel any abnormality in the diseased organ, and this is often only diagnosed by examining microscopically the expressed products for pus and organisms. Even when the vesiculæ seminales are closed, perseverance in massage is the best treatment, and the operations vasostomy and vesiculotomy are never necessary.

Chronic cowperitis is often the cause of recurrences in gonorrhœe. It is much more common than is generally supposed, and can be diagnosed in 5 per cent. of cases of gleet. The treatment is massage, first once a week, then twice a week. The forefinger should be passed into the rectum and the thumb placed on the perineum on either side or the median raphé, when it will be an easy matter to palpate the gland between the thumb and forefinger. The enlarged gland does not feel at all unlike a malignant lymphatic gland.

An infected para-urethral canal, often seen in cases of hypospadias, is easily destroyed with the electric cautery, and an infected para-urethral gland by injecting $1 \mathrm{~m}$. of pure carbolic into the body of the gland. A wart growing from the mucous membrane of the meatus occasionally causes a gleet and is easily destroyed by applying trichloracetic acid roo per cent.

During the treatment of gleet, it is not necessary to adhere so rigidly to the strict dietetic and hygienic measures which are prescribed for acute gonorrhœea. The diet may be more liberal, and a moderate amount of exercise may be taken: If the patient leads a healthy life and raises his resistance as much as possible, he will help considerably to administer the final knock-out blow to the infection.

\section{FOR NOTES.}

